\title{
Butyrate boosts microbicidal macrophages
}

Bacterial fermentation products such as short-chain fatty acids (SCFAs) are important mediators of the crosstalk

butyrate imprints a noninflammatory antimicrobial programme in gut macrophages

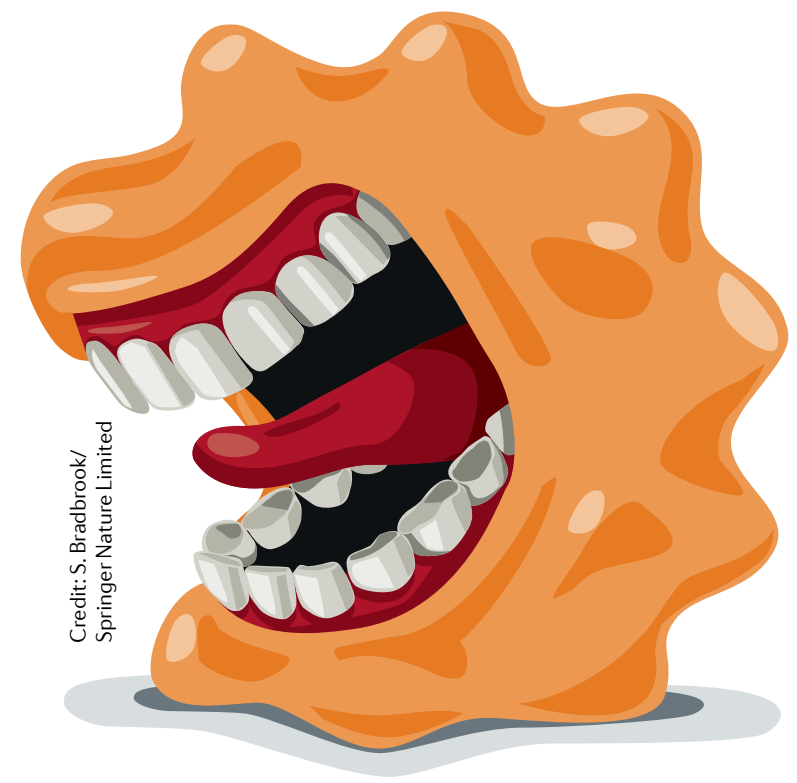

Cells incubated with butyrate showed superior killing of Gram-negative bacteria, such as Salmonella enterica or Citrobacter rodentium, as well as Gram-positive bacteria such as Staphylococcus aureus.

Metabolomic analysis of butyratetreated macrophages revealed a substantial reduction in glycolysis. This was associated with higher amounts of AMP, an inducer of AMP kinase, which, in turn, inhibits mechanistic target of rapamycin (mTOR). As mTOR is a positive regulator of glycolytic enzymes, its inhibition may explain the observed reduction in glycolysis.

mTOR inhibition was important for microbicidal functions in butyratetreated macrophages, as treatment with an mTOR activator blunted their antibacterial activity. Since mTOR is known as a central regulator of autophagy, the authors tested whether the enhanced antimicrobial activity was associated with the LC3 protein, a central player in the autophagy pathway. Indeed, they observed higher LC3 turnover and enhanced generation of reactive oxygen species (ROS), an antimicrobial effector mechanism associated with LC3-mediated phagocytosis.

Further analysis by single-cell RNA sequencing revealed a butyrateinduced antimicrobial signature that was characterized by increased expression of genes such as $S 100 A 8$ and S100A9. These encode calprotectin, a protein with antimicrobial function. Knockdown of S100A8 and S100A9 with small interfering RNA impaired macrophage microbicidal function. These results indicate that butyrate induces a synergistic programme of LC3-associated host defence and the production of antimicrobial peptides.

Since butyrate is known as an HDAC inhibitor, the authors speculated that it exerts its functions through the induction of epigenetic changes. Indeed, they found that other HDAC inhibitors, such as valproate and SAHA, can also increase the expression of S100A8 and enhance the antimicrobial function of macrophages. Further analysis showed that butyrate appears to act via HDAC3 and that inhibition of HDAC3 is sufficient to induce the differentiation of macrophages with enhanced microbicidal functions. This indicates that the inhibition of HDAC3 is upstream of metabolic changes and antimicrobial responses in butyrate-treated macrophages.

To investigate whether these observations are of relevance in vivo, mice were given a daily oral dose of either butyrate or water for 1 week. At this point, colonic macrophages were harvested and subjected to bacterial killing assays. Analogous to the observations with human butyrate-treated macrophages, colonic macrophages from butyrate-treated mice had enhanced antimicrobial activity. This appeared to be mediated by local effects, as macrophages differentiated from the bone marrow of butyrate-treated or control mice showed no differences in antimicrobial potency. When challenged with either Salmonella or $C$. rodentium, butyrate-treated mice showed significantly reduced bacterial dissemination.

These results support a model in which butyrate imprints a non-inflammatory antimicrobial programme in gut macrophages, which is consistent with the concept that butyrate-producing commensal bacteria shape host-microbial crosstalk to promote a stable relationship. This has implications for the prevention and therapy of disorders associated with intestinal inflammation such as IBD.

Alexandra Flemming

ORIGINAL ARTICLE Schulthess, J. et al. The short chain fatty acid butyrate imprints an antimicrobial program in macrophages. Immunity https://doi.org/ 10.1016/j.immuni.2018.12.018 (2019) 